\title{
Exosomes - a potential indicator and mediator of cleft lip and palate: a narrative review
}

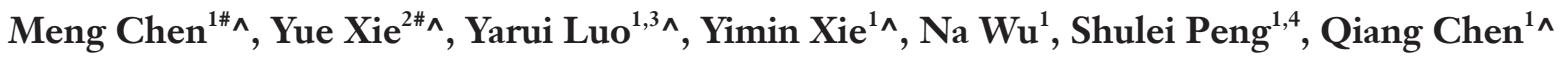 \\ ${ }^{1}$ Department of Paediatric Surgery, Chongqing University Three Gorges Hospital, Chongqing, China; ${ }^{2}$ Department of Burn and Plastic Surgery, \\ Children's Hospital of Chongqing Medical University, Chongqing, China; ${ }^{3}$ Department of Outpatient, Chongqing University Three Gorges \\ Hospital, Chongqing, China; ${ }^{4}$ Department of Sleep Medicine Centre, Chongqing University Three Gorges Hospital, Chongqing, China \\ Contributions: (I) Conception and design: S Peng, Q Chen; (II) Administrative support: All authors; (III) Provision of study materials and figures: M \\ Chen, Y Xie, Y Luo; (IV) Collection and assembly of data: Y Luo, N Wu; (V) Data analysis and interpretation: All authors; (VI) Manuscript writing: \\ All authors; (VII) Final approval of manuscript: All authors. \\ \#These authors contributed equally to this work. \\ Correspondence to: Shulei Peng. Department of Sleep Medicine Centre, Chongqing University Three Gorges Hospital, No. 862 North Mountain Road, \\ Wanzhou District, Chongqing 404000, China. Email: 1641070090@qq.com; Qiang Chen. Department of Pediatric Surgery, Chongqing University \\ Three Gorges Central Hospital, No. 165 Xincheng Road, Wanzhou District, Chongqing 404000, China. Email: cqwzchenqiang@163.com.
}

Objective: This article summarizes the recent literature on noncoding ribonucleic acids (ncRNAs) in relation to cleft lip with or without palate and exosomes and their usage in craniofacial diseases.

Background: Cleft lip with or without cleft palate $(\mathrm{CL} / \mathrm{P})$ is a common congenital malformation with genetic and environmental risk factors that affects numerous children and families. Surgical procedures can correct deformations; however, residual sequelae remain after surgery. Studies exploring the pathogenesis of $\mathrm{CL} / \mathrm{P}$ are crucial for its early diagnosis and treatment and can inform treatment strategy decisions, etiology searches, and treatment during pregnancy. Recently, research has shown that most disease-related genes are ncRNAs, which are important transcripts in the human transcriptome. ncRNAs include microRNAs, long noncoding RNAs, and circular RNAs. These ncRNAs play essential roles in various pathophysiological processes, including cell proliferation, migration, apoptosis, and epithelial-mesenchymal transition. Previous studies on protein-coding genes have identified a number of genes related to CL/P; however, the pathogenesis of CL/P has not yet been thoroughly explained. Exosomes are vehicles that transfer various bioactive molecules between cells and represent a new method of intercellular communication. Research has shown that exosomes are related to some craniofacial diseases.

Methods: We searched the PubMed database for recently published English-language articles using the following keywords: "cleft lip with or without palate," "noncoding RNA," "exosomes," and "craniofacial diseases". We then reviewed the retrieved articles.

Conclusions: As exosomes serve as cellular communicators and the palate consists of epithelial and mesenchymal cells, communication between the two cell types may affect its formation. Thus, exosomes could represent a new indicator and mediator of CL/P.

Keywords: Exosome; Cleft lip with or without cleft palate (CL/P); circle RNAs (circRNAs); long noncoding RNAs (lncRNAs); microRNAs (miRNAs)

Submitted Jul 23, 2021. Accepted for publication Sep 07, 2021.

doi: $10.21037 /$ atm-21-4198

View this article at: https://dx.doi.org/10.21037/atm-21-4198

^ ORCID: Meng Chen, 0000-0003-2722-9940; Yue Xie, 0000-0002-9831-9567; Yarui Luo, 0000-0002-0612-5993; Yimin Xie, 0000-0001-65553667; Qiang Chen, 0000-0002-4312-1879. 


\section{Introduction}

Cleft lip with or without cleft palate $(\mathrm{CL} / \mathrm{P})$ is a worldwide congenital deformation disease that affects thousands of children and families. CL/P can be classified according to genotypes. Specifically, syndromic CL/P results from gene mutations or chromosomal abnormalities, while nonsyndromic $\mathrm{CL} / \mathrm{P}$ is a consequence of multiple factors, including environmental and genetic factors. Any temporal, spatial, or quantitative dysregulation of molecular signal transmission during labial and palatal morphogenesis, growth, and fusion can lead to CL/P. With the development of genomics, researchers have focused on CL/P-related genes. According to Manolio's genome-wide association study, $80 \%$ of the genes related to CL/P are located outside protein-coding loci; these genetic molecules regulate gene expression at the posttranscriptional level via gene silencing and are closely related to biological events, such as cell proliferation, apoptosis, adhesion, and epithelial-mesenchymal cell transdifferentiation. Generally, noncoding ribonucleic acids (ncRNAs) have been shown to have a significant role in the occurrence of $\mathrm{CL} / \mathrm{P}$ $(1,2)$. Previous genetics of $C L / P$ were focused on singletype cells, such as mesenchymal cells or epithelial cells. Secondary palate development requires the cooperation of both types of cells. There are no studies that present whether there is a mutual adjustment relationship between them and how to contact them. First identified in the late 1980s, exosomes are a subset of extracellular vesicles secreted by most cells that were originally thought to carry cellular waste. However, further research revealed that exosomes represent a significant and novel model of intercellular communication and contribute to numerous biological and pathological processes, including craniofacial diseases (3). Exosomes contain a variety of cargos, such as lipids, proteins, metabolites, and nucleic acids, which can be transported to target cells. Promising research suggests that exosomes could serve as prognostic biomarkers and therapeutic targets in many diseases (3). Thus, exosomal commerce is a promising emerging industry that could contribute to the medical armamentarium. We will review the possible potential applications of exosomes in cleft lip and palate and hope that new discoveries will be made in disease prevention and treatment in the future.

We present the following article in accordance with the Narrative Review reporting checklist (available at https:// dx.doi.org/10.21037/atm-21-4198).

\section{Methods}

We searched the PubMed database for recently published English-language articles using the following keywords: "cleft lip and palate," "noncoding RNA or miRNA, lncRNA, circRNA," "exosomes," and "craniofacial diseases." We then reviewed the retrieved articles.

\section{Results}

\section{$C L / P$ and related genes and molecular basis}

With a prevalence of $0.775-1.089 \%$ among live births, $\mathrm{CL} / \mathrm{P}$ is one of the most common postnatal craniofacial defects and congenital malformations for which there are ethnic, racial, and geographic variations (4). Approximately $70 \%$ of CL/P cases are sporadic (nonsyndromic), while the remaining $30 \%$ comprise syndromic phenotypes with Mendelian traits that result from a single gene mutation or chromosomal abnormalities $(5,6)$. Both environmental and genetic factors play a crucial role in nonsyndromic CL/P. Labial and palatal development is regulated by gene-controlled cellular signal transmission and conduction. Based on genome-wide association study (GWAS) data, the human PPI network, and differential gene expression profiles, Yan et al. identified a total of $87 \mathrm{CL} / \mathrm{P}$-associated genes of both European and Asian ancestries in dmGWAS, which included nine novel CL/P candidate genes (BRD1, CREBBP, CSK, DNM1L, LOR, PTPN18, SND1, TGS1, and VIM). Functional enrichment analysis demonstrated that these genes were involved in cell adhesion, cell localization to the plasma membrane, regulation of the apoptotic signaling pathway, and other pathological conditions (4). Early genome scans provided suggestive evidence for CL/P. A meta-analysis that included 13 scans with data from several regions demonstrated strong evidence that nonsyndromic CL/P (NSCL/P) was associated with $9 \mathrm{q} 21$, at which key candidate genes were related to patched (PTCH1), receptor tyrosine kinase $(R T K)$-like orphan receptor 2 (ROR2), transforming growth factor-beta receptor 1 (TGFBR1), and forkhead box E1 (EOXE1) (7). Other GWAS also identified other important loci involved in NSCL/P, including a strong association with the genomic region at 8q24.21 (8). Syndromic CL/ Ps are more often attributed to one congenital cause or disrupted gene than NSCL/P. The underlying etiologies associated with an orofacial cleft phenotype may be difficult to determine because the role of specific genes in CL/ 
GD13.5

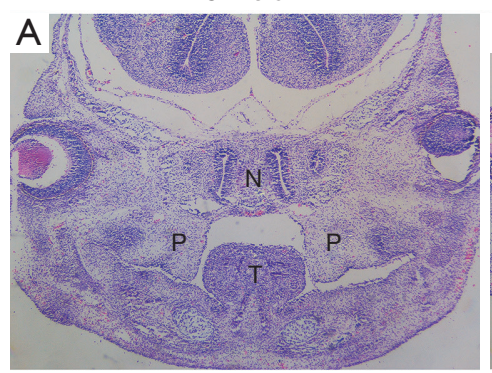

GD14.5

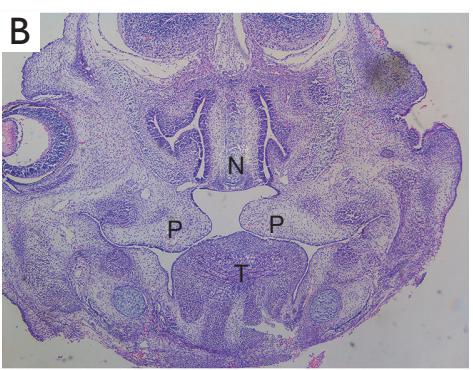

GD15.5

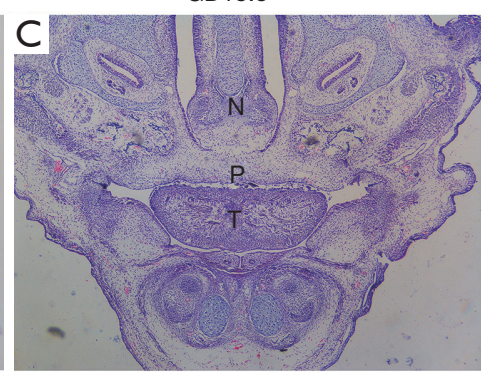

Figure 1 Representative histological frontal sections from the middle regions of the developing palate at different gestation days (H\&E staining, $\times 100$ ). (A) At GD13.5, the palatal shelves exhibited distinct shapes along the AP axis; (B) at GD14.5, the palatal shelves had elevated to the horizontal position; (C) at GD15.5, the palatal shelves had fused at the middle region with complete removal of the midline epithelial seam. N, nose; P, palate; T, tongue; GD, Gestation days.

P-associated cases is hard to describe thoroughly, as multiple disease-causing genes and factors can exist, especially if $\mathrm{CL} / \mathrm{P}$ is a minor feature and is not the primary focus of studies on a particular condition. Reynolds et al. reviewed and demonstrated genetics associated with syndromic CL/ $\mathrm{P}$, including Pierre Robin Sequence, Popliteal Pterygium Syndrome, Rapp-Hodgkin Syndrome and so on (9). After GWAS, some new technologies have gradually matured, such as exome sequencing, copy number variation (CNV) and whole-genome sequencing studies (WGS). Younkin et al. used CNV to discover genes with research potential, such as ch7p14.1, AD-AM3A, MGAM and ADAM5A (10).

\section{An overview of palatogenesis}

Based on the palatogenesis process in mouse models, which precisely mirrors that in humans, researchers have examined the mystery behind this elaborate developmental process. In mammals, the formation of 5 facial prominences surrounding the primitive mouth (i.e., the frontonasal prominence on the rostral side, a pair of maxillary prominences laterally, and a pair of mandibular prominences caudally) triggers facial development. As the embryo develops in the fifth week, palate formation begins in two regions: the primary and secondary palates. The primary and secondary palates form as outgrowths from the oral side of the medial nasal processes, which are derived from frontonasal prominence, and maxillary processes, which are formed by maxillary prominence (see Figure 1). In a process known as palatal shelf elevation, the secondary palatal shelves initially grow vertically, flanking the developing tongue, and subsequently reorient to a horizontal position parallel to the tongue. Ultimately, the paired secondary palatal shelves extend toward the midline and fuse with each other, forming a complete palate (11). The palates are elevated and move toward each other. Each palatal shelf comprises medial edge palatal mesenchymal (MEPM) cells, which mainly originate from the neural crest and a surrounding undifferentiated oral epithelium. During the fusion of the palatal shelves, a midline epithelial seam is formed via the adhesion of the medial edge epithelia (MEE) from each palatal shelve, which subsequently disappears to allow for the confluence of MEPM cells (12). In addition, the intact roof of the oral cavity is completed when the secondary palate fuses anteriorly with the primary palate and anterodorsally with the nasal spectrum at 12 weeks of gestation in humans (and embryo day 17 in mice).

Along the anterior-posterior axis of the palate, molecular heterogeneity has been shown to be differentially expressed in mouse models (13). Msx 1, Bmp4, and Bmp2 in the bone morphogenetic pathways; Shb and Spry 2 in the sonic hedgehog (Shh) signaling pathways; FgflO and Fgf7 in the Fgf signaling pathways; and the Shox2 network, Efnb1 in Ephrin signaling and the Tgf- $\beta$ family are associated with the anterior palate. The genes related to the posterior palate are Meox2, Tbx22, and Barx1. Mutations of MSX1 in humans have been associated with isolated nonsyndromic cleft palate (14).

\section{Epithelia-mesenchymal interaction during palatogenesis}

Mesenchymal cells with an irregular, elongated, and spindle-like morphology possess front-to-back polarity that enables migration and invasion. Epithelial cells have flat and polygonal shapes, neatly stick to the basal membrane from cohesive cell layers to intercellular junctions, and 
possess limited mobility. The removal of MEE cells is crucial for palatal fusion. Among the controversial theories on the mechanism underlying the disappearance of MEE, 3 major theories have dominated: (I) epithelialmesenchymal transdifferentiation in which epithelial cells acquire the morphology and migratory and invasive capacities of mesenchymal cells $(15,16)$; (II) MEE cell apoptosis (17); and (III) the lateral migration of MEE cells (18). Mesenchymal cell proliferation, migration and apoptosis are closely associated with palate elevation, augmentation, and convergence. Recent studies have shown that the development of the palatal shelves is controlled by reciprocal epithelial-mesenchymal interactions and molecular mechanisms (19). The disruption of palatal mesenchymal smoothened (Smo) function, which encodes the transmembrane protein required for transducing sonic hedgehog (Shh) signaling, indirectly affects palatal epithelial cell proliferation (20). Interestingly, Smo function is not required in the epithelium for palatal outgrowth (21), which suggests a potential mesenchymal-epithelial interaction.

Molecular pathways are interrelated and mediate communication between the mesenchyme and the epithelium; for instance, the BMP and FGF signaling pathways both use Shh signaling in the anterior palate to regulate palatal growth (22). Msx 1 expression in the anterior mesenchyme regulates Bmp4 expression, which regulates $S h b$ expression in the epithelium. Shb in turn regulates $B m p 2$ expression in mesenchymal cells, while $B m p 2$ expression regulates mesenchymal cell proliferation (14).

Homozygous mice with a null mutation in either fibroblast growth factor 10 (Fgf10) (an essential mesenchymal signal) or the encoding gene of its receptor, fibroblast growth factor receptor $2 \mathrm{~b}$ (Fgfr2b), exhibited cleft palate (23). Fhf10 messenger RNA (mRNA) is expressed restrictively in mesenchymal cells, while Fgfr $2 \mathrm{~b}$ mRNA is abundantly detected in the overlying epithelium. When both epithelial and mesenchymal proliferation were reduced in the absence of Fgf10 and Fgfr2b, Shh expression decreased dramatically in epithelial Fgf10 $0^{-/-}$and Fgfr $2 \mathrm{~b}^{-/-}$ embryos, suggesting that the proliferation of mesenchymal cells in mutant mice might be a consequence of the reduction in Shh expression in the epithelium (21).

\section{$n c R N A$ in $C L / P$}

As their name suggests, ncRNAs are RNAs without protein-coding potential and account for the majority of RNAs. There is increasing evidence that ncRNAs have a huge effect on molecular mechanisms and organogenesis, including palate formation (see Figure 2). They can be divided into two subclasses according to size: (I) long noncoding RNAs (lncRNAs); that is, ncRNAs consisting of more than 200 nucleotides (nt); and (II) small or shortened ncRNAs; that is, ncRNAs consisting of less than $200 \mathrm{nt}$, which include microRNAs (miRNAs) with a size of $20 \mathrm{nt}$, and circular RNAs (circRNAs). Many miRNAs have already successfully served as biomarkers or therapeutic targets for a wide range of diseases and represent a new field for disease diagnosis and treatment (24).

\section{The role of miRNA in CL/P and palatal cellular comportments}

miRNAs act as gene expression regulators that reduce the stability of the target gene and inhibit its translation by binding to a complementary sequence in the 3' untranslated region (UTR) of a target messenger RNA (mRNA) (25). miRNAs consistently interact with proteins to regulate RNA transcription, processing, localization, function and degradation. Emerging evidence indicates that miRNAs are related to palate proliferation, migration, and fusion (5). The deletion of any homozygous gene in mice causes severe growth retardation and embryonic death after embryo implantation, indicating that miRNAs have an important role in embryonic growth and development (26), including palatal development. miRNAs play different roles in the development of different tissues, some of which are related to congenital defects (27). The expression levels of plasma miRNAs were found to differ between nonsyndromic cleft palate and nonsyndromic cleft lip with palate (CLP), and the expression level of 305 miRNAs in plasma from patients with CL/P and 241 miRNAs in plasma from patients with CLP differed from those in the control group (28).

The overexpression of miR-140-5p, which plays a critical role in palate morphogenesis through the suppression of platelet-derived growth factor receptor alpha (PDGFRA) in zebrafish, was shown to inhibit the expression of BMP2 and FGF9 in human palatal cells and the expression of FGF9 and PDGFRA in mouse palatal cells (29). miR-200b was shown to regulate the Smad2, E-cadherin and Snail genes during palate development in mice. The ectopic expression of the miR-200b gene led to a disturbance in the removal of MES, and the overexpression of miR-200 proliferated the epithelium in MES and mesenchyme around the remaining MES (30).

As a gene cluster located on human chromosome $13 \mathrm{q} 31.3$, the miR-17-92 miRNA cluster is regulated by 


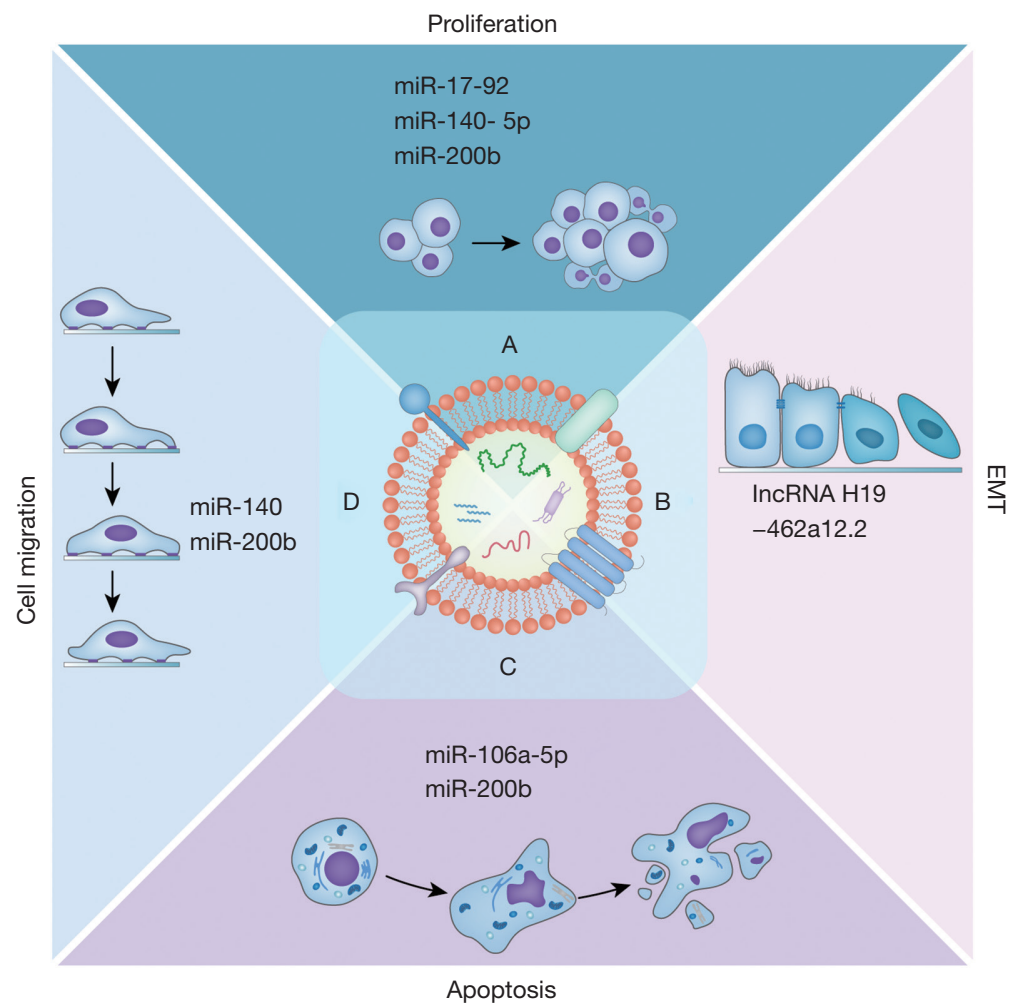

Figure 2 The physiological functions of ncRNAs in CL/P. (A) miR-17-92, miR-140-5p, and miR-200b are involved in cellular proliferation; (B) lncRNA H19-462a12.2 is involved in EMT; (C) miR-106a-5p and miR-200b are involved in apoptosis; (D) miR-140 and miR-200b are involved in cell migration. EMT, epithelial-mesenchymal transition.

bone morphogenetic protein (BMP) signaling, whose deficiency in mice and humans has been shown to cause $\mathrm{CL} / \mathrm{P}$ and other craniofacial anomalies (31). It is also controlled by AP-2 $\alpha$, for which mutant mice have CL/P (31). Fgf signaling, whose deficiency results in cleft palate, was revealed to be regulated by miR-17-92 (31). Embryos carrying a miR-17-92 mutation exhibit severe defects in the middle face, such as mandibular hypoplasia and CL/P (32). miR-17-92, whose expression was found to be decreased in palatal shelves and palatal mesenchyme from E12 to E14, promoted MEPM proliferation and collagen synthesis by inhibiting the transforming growth factor beta 1 (TGF- $\beta 1$ ) signaling pathway (32).

miR-106a-5p has been shown to induce palate mesenchymal cell apoptosis and regulate cholesterol metabolism, which can affect cell proliferation via the TGF- $\beta /$ Smad/signaling pathway, which in turn can lead to palatal tissue abnormalities in mice following RA-induced cleft palate (33). However, to date, only a few miRNAs have been proven to function in palatal development.
Investigating miRNAs related to CL/P could help promote their usage as biomarkers and therapeutic targets and inform prenatal screening and treatment. The occurrence of diseases will cause more frequent signal delivery, so the content of exosomes will increase and can be detected more easily. In addition, the molecules related to maternal cell status in exosomes are protected by a membrane. Information about the state change of the maternal cell is directly transmitted to the recipient cell and to regulate its changes. Therefore, miRNAs in exosomes in the plasma are more helpful for us to study the etiology of cleft lip and palate deformity.

\section{LnRNA in CL/P and palatal cellular comportments}

LncRNAs are involved in molecular mechanisms. Specifically, lncRNAs regulate transcription, influence mRNA stability, and regulate protein function $(34,35)$. Recently, lncRNAs have been shown to be involved in many physiological and pathological processes, such as the immune response, tumor metastasis, cell differentiation, and cell 
cycle regulation (36). Salmena's research group proposed the competing endogenous RNA (ceRNA) hypothesis, which conjectured that lncRNAs could "sponge" miRNAs with miRNA response elements (MREs) to bate their function on mRNA (37). Shu et al. analyzed lncRNAs in the RNA sequencing map of all-trans retinoic acid-induced embryonic mice, constructed a lncRNA-related ceRNA network, verified the expression profile of lncRNAs via quantitative polymerase chain reaction, and determined that the NONMMUT004850.2/NONMMUT024276.2-miR-741$3 \mathrm{p} / \mathrm{miR}-465 \mathrm{~b}-5 \mathrm{p}-$ Prkar1 aceRNAs network might be related to $\mathrm{CL} / \mathrm{P}$, as conjectured by the ceRNA hypothesis (38).

Gao et al. used a high-throughput screen to identify 26246 new lncRNAs and 9635 known lncRNAs in rats with 2,3,7,8-tetrachlorobenzo-p-dioxin (TCDD)-induced cleft palate and found that 413 lncRNA transcripts differed significantly from those in the control group (39). Gao et al. also identified 7 lncRNAs that might target Smad1 and Smad5, which are key molecules in the BMP signaling pathway, and showed that lncRNAs might be related to BMP signal transduction in TCDD-induced cleft palate (39). In addition, other studies have shown that the expression levels of LEF1, NONMMUT34790.2, and SMAD7 are reduced in mice with clef palate. Researchers have postulated that these genes inhibit EMT of palatal epithelial cells during palatal fusion through a "IncRNAtranscription factor (TF)-target gene" negative regulatory mechanism (12). lncRNA H19 acts as a ceRNA for miR138 and miR-200a, antagonizing their function, which in turn leads to the endogenous inhibition of the core marker genes ZEB1 and ZEB2 in targeted mesenchymal cells (40). Thus, lncRNA H19 appears to be involved in and regulate the EMT process.

\section{CircRNAs in CL/P and palatal cellular comportments}

CircRNAs have a covalently closed-loop structure without 5'-3' polarity and a polytail, which is generated from reverse splicing between specific and conserved sequences in upstream and downstream regions. The known functions of circRNAs include the preservation of miRNAs or proteins, the regulation of transcription, interference with splicing, and even the transcription of peptides; however, the functions of most circRNAs remain unknown (41). Researchers have identified additional circRNAs that participate in important pathophysiological processes, such as the dynamic regulatory effects of selective splicing factor Quaking (QKI) during EMT in humans. Notably, OKI itself is also regulated during EMT (42), which is an important mechanism by which palatal epithelial cells disappear, allowing the palates to fuse. Based on the construction and functional analysis of circRNArelated networks and in accordance with the hypothetical relationship between ceRNAs and the palate, Shu et al. found that the circRNA 0954-miRNA-881-3p-prkar1 ceRNA network is a potentially important mechanism related to the dysfunction of palatal fusion (43).

\section{Prenatal approaches to CL/P: Diagnostic screening and targeted molecular therapeutics}

As the primary and secondary palates form between 6-7 and $8-10$ weeks of gestation, respectively, cleft examination before or during this period is vital to maximize therapeutic effects. Conventional ultrasonography can be used to detect the maxillary lip; however, spatially, the palatal cleft is left unevaluated. Laboratory examinations should be undertaken to evaluate palatal dysmorphology during pregnancy. Some prenatal screenings adopt routine invasive approaches, including harvesting fetal cells [prenatal cell-free deoxyribonucleic acid (cfDNA) and cfRNA via real-time ultrasound-guided transabdominal aspiration of amniotic fluid at week 16 of gestation and sampling placental cells retrieved between 10-13 weeks of gestation. Due to the damage of invasive examinations, cell-free nucleic acids could be harvested from maternal blood to diagnose cleftassociated syndromes (44). Furthermore, this recent noninvasive approach prevents fetal loss (44). A microarraybased cytogenic technique can also detect aneuploidy and provide additional genetic information and is preferred in clinical practice, as it has a higher sensitivity and shorter turnaround times than conventional karyotyping (45). Fetal-parental-trio whole-exome sequencing (WES) with cleft detection is a next-generation technology related to a cytogenic or microarray technique that can increase the detection of fetal structural anomalies (46). WES research has harvested fetal tissue through invasive methods, including amniotic fluid, chorionic villus sampling, or fetal blood; however, less invasive strategies for obtaining fetal tissue, such as the genetic analysis of fetal cfDNA and cfRNA, should be employed to promptly detect dysmorphology in offspring. To implement cell-free fetal transcriptomics, which is a prospering diagnostic tool, reference data of key genetic expressions across a range of developmental stages need to be acquired, and accurate determinations of gestational age need to be made (47).

Research has shown that palatal morphogenesis can 


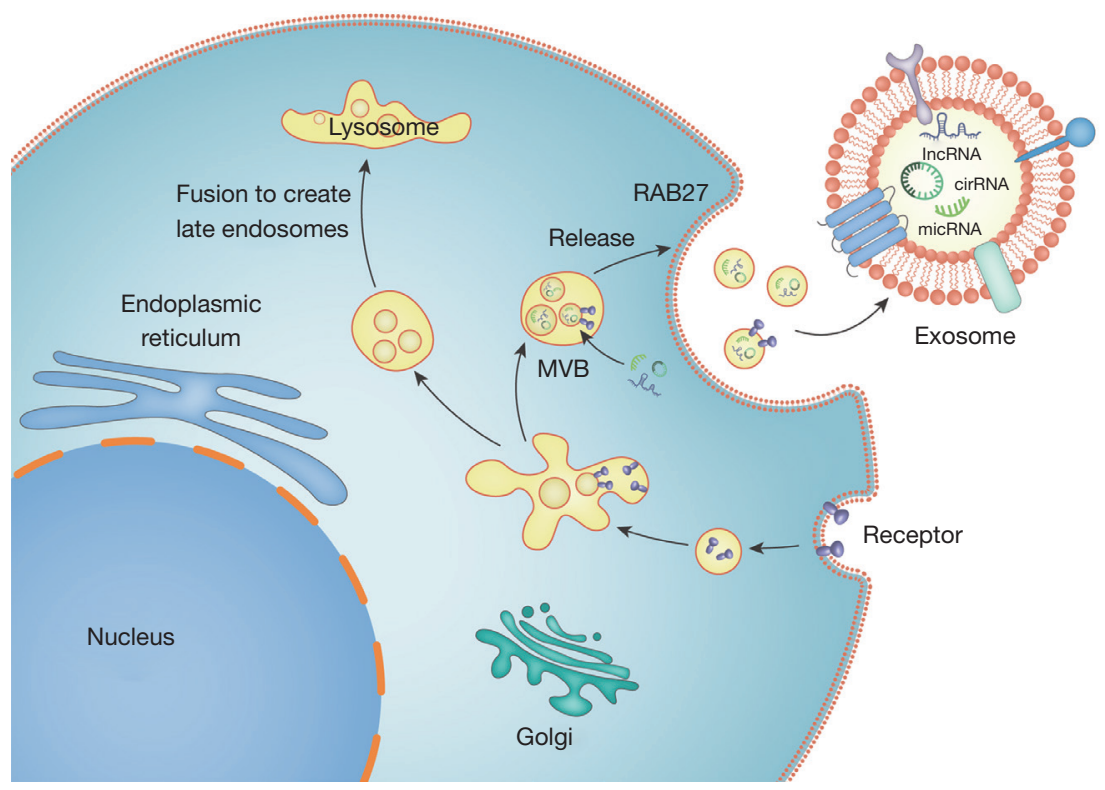

Figure 3 The biogenesis and release of exosomes. First, early endosomes form through endocytosis via the plasma membrane. Second, early exosomes maturate into MVBs via endocytosis. MVBs either fuse with lysosomes for degradation or fuse with plasma membranes. The secreted exosomes contain lncRNAs, circRNAs, and miRNAs, which come from parental cells. MVBs, multivesicular bodies.

be restored during pregnancy (15). Precision therapies for Mendelian diseases, such as those that directly target disease-related pathways or influence the expression of disease-related genes, are clinically available for many conditions, such as lysosomal storage disorders, cystic fibrosis, and spinal muscular atrophy (48). Such approaches have been applied to monogenic conditions. The extension of these approaches to conditions for which there is genetic heterogeneity but pathway homogeneity is logical. Unlike inherited metabolic diseases that require chronic treatments with repeated infusions to replace bioactive molecules and postnatal surgical interventions that require a long operative period but for which physiological and physical sequelae occur, prenatal therapies can affect nonreversible developmental stages. Indeed, these short-term treatments can produce permanent effects. Targeting interrelated genes may have therapeutic benefits for cleft patients with a range of genetic etiologies. A wide range of causative mutations are implicated in CLP. An understanding of CL/P-related genes and pathways illuminates potential therapeutic targets. The highly targeted and efficient delivery of therapeutic factors could dramatically facilitate treatment efficiency.

Extensive gene regulatory networks consisting of multiple signaling pathways and transcription factors are involved in the control of palatal shelf growth, patterning, and fusion (19). Any temporal, spatial, or quantitative dysregulation of molecular signal transmission during labial and palatal morphogenesis, growth, and fusion can lead to CL/P. Exosomes, which are communication messengers between cells, could be abnormal due to external factors, which could in turn lead to CL/P.

\section{Exosomes: an arsenal of ncRNAs serve as Indicators and Mediators of Diseases}

\section{Exosomes}

Exosomes are membrane-enclosed microvesicles that are actively secreted by cells $(49,50)$. Exosomes have a diameter of 40-200 nm, a density of $1.13-1.18 \mathrm{~g} / \mathrm{mL}$, and a cup-like shape, as visualized by electron microscopy $(49,50)$. The biogenesis of exosomes begins with the endosomal pathway; early endosomes form through endocytosis via the plasma membrane and then maturate into multivesicular endosomes (MVEs) via endocytosis. MVEs fuse with the plasma membrane and are secreted into the extracellular space as exosomes (see Figure 3). Exosomes can selectively envelop biological molecules according to their parental cells and pathophysiological status and thus function as transporters among cells (51). As exosomes participate in fundamental 
physiological processes or pathological disorders with their cargos, their effects can be beneficial or adverse according to their cargos. An exosomal membrane has a phospholipid bilayer structure and topological protein structure similar to that of a plasma membrane, which not only protects the contained molecules from proteases and nucleases but also mediates signal transduction via cell surface signaling molecules (52). Exosomes deliver the genetic information that they carry by fusing with nearby cells or other cells that they reach by circulating in the bloodstream. Exosomes contain an abundance of biological molecules (9,769 proteins, 3,408 mRNAs, and 116 lipids were listed in the latest exosome database) (53) and are especially rich in ncRNAs (see Figure 2). According to an RNA sequencing study, the amount and type of RNA contained in exosomes differs from that in parental cells, which suggests that exosomal RNAs are specifically packaged (54).

\section{Exosomal function on cells and epithelium-mesenchymal communication}

Exosomes contain a wide spectrum of molecules characterized by parental cells, which can be transported between cells under the protection of a lipid bilayerenclosed envelope. Mittelbrunn et al. concluded that exosomes may contain many disease-related ncRNAs that could be altered when parental cells are stimulated by external factors (55). Exosomes appear to be involved in EMT, which also occurs in palatal epithelium during cleft fusion in tumoral pathological processes (56). In pancreatic ductal carcinoma (PDAC), exosomal Sox2ot, which was identified from highly invasive PDAC cells, works as a ceRNA that can regulate SOX2 expression responsible for increasing EMT, leading to the promotion of invasion and metastasis of PDAC (57). The presence of caveolin-1 in prostate cancer-derived exosomes was shown to effectively induce the EMT process via nuclear factor kappa-lightchain enhancer of activated $\mathrm{B}(\mathrm{NF}-\kappa \mathrm{B})$ signaling (58). Exosomes derived from hepatocellular carcinoma cells could regulate EMT by activating the TGF- $\beta /$ Smad signaling pathway, which has also been shown to be involved in apoptosis and metabonomic changes in cleft palate (33), which in turn could promote the migration and invasion of target cells (59). Interestingly, hypoxia is also involved in the regulation of EMT by exosomes. Exosomes derived from hypoxic marrow-derived mesenchymal stem cells (MSCs) mediate the transfer of miRNAs from bone marrowderived MSCs to lung cancer cells, stimulating STAT3 phosphorylation and thus activating STAT3 signalinginduced EMT and promoting the invasion abilities of lung cancer cells (60). To date, no studies have explored the relationship between exosomes and EMT of the palatal epithelium; however, exosomes may be involved in EMT through the same signaling pathway as that in tumorigenesis during palatal fusion.

There is evidence that exosomes transport between the epithelium and mesenchyme (61). Jiang et al. found that exosomes were preferentially endocytosed by reciprocal cell type epithelium and mesenchyme, affecting cell differentiation and matrix synthesis during organ development (62). Hayashi et al. found that mesenchymal exosomal miRNAs mobilized genetic signals in the epithelium and specifically influenced progenitor cell expansion during organogenesis (63).

Exosomes are ideal noninvasive diagnostic biomarkers, have virtues that are specific to parental cells, display a high surface viscosity and rigidity due to the lipid component of their membranes, have agglutination abilities due to their surface antibodies, and are a stable biological fluid. Research indicates that exosomes might be effective diagnostic and therapeutic tools for diseases such as cancer, rheumatoid arthritis, myocardial infarction, oral disease, and pregnancy disorders.

\section{Applications of exosomes in craniofacial diseases and prenatal screening}

Research has shown that the reduction of CD9/CD81 exosomes in saliva is associated with the pathogenesis of periodontal diseases (64). A comparison between salivary exosomes in young adults with severe periodontitis and healthy individuals via mass spectrometry and a gene ontology analysis revealed that 26 immune-related proteins were specific to severe periodontitis (65). Additionally, exosome-associated nucleic acids could serve as potential biomarkers of periodontal diseases. One study, which used atomic force microscopy to collect exosomes from saliva, reported that the morphological characteristics of exosomes differ between patients with oral cancer and healthy individuals (61). Thus, oral cancer exosomes can be accurately selected and distinguished from their benign counterparts by detecting subtle changes in the conformations of proteins, lipids, and nucleic acids. The proteomic analysis of the protein carried in exosomes also provides a functional diagnostic tool for the detection of malignant transformations in oral cancers (66). Xie et al. 


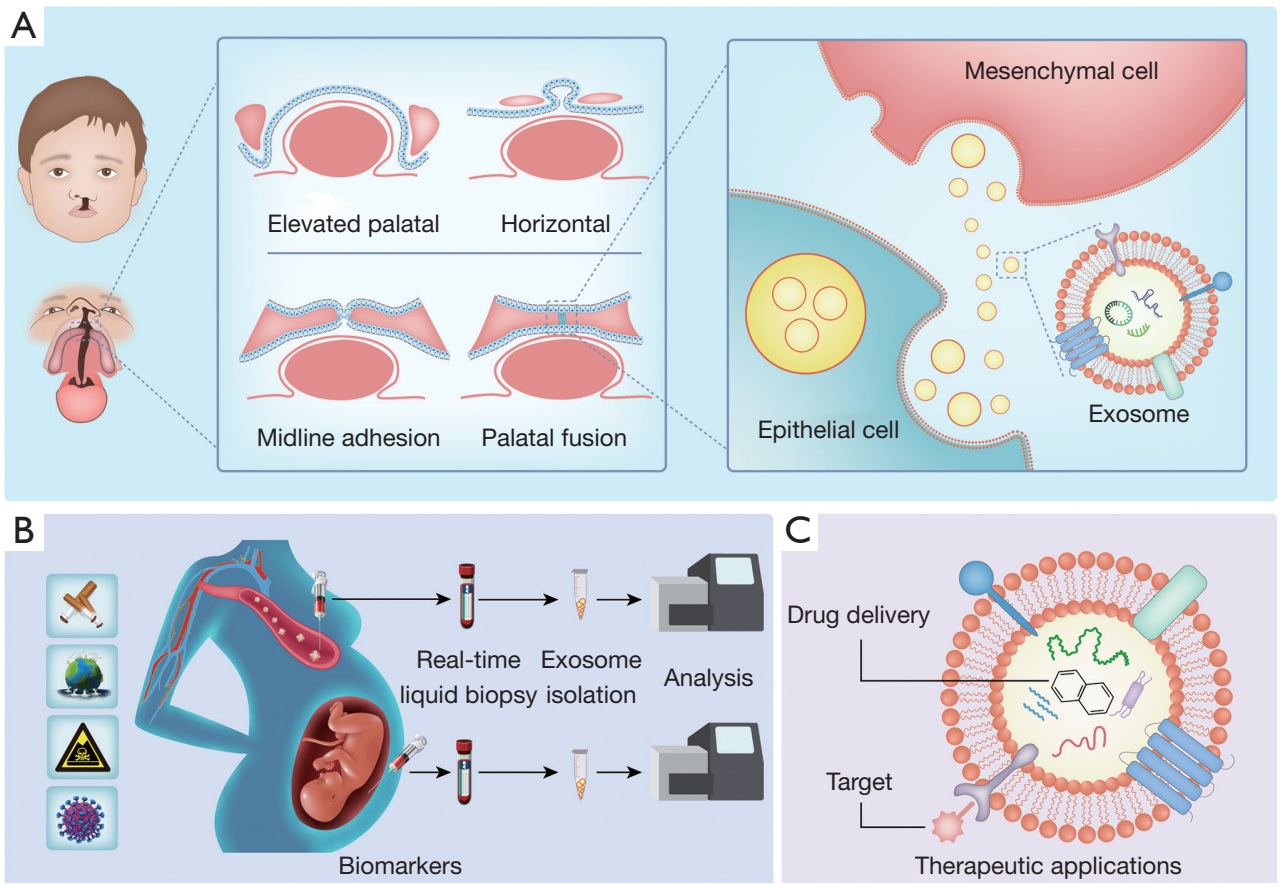

Figure 4 Potential clinical applications of exosomes in CLP. (A) Mesenchymal cells and epithelial cells, which participate in palatal fusion, communicate with each other through exosomes, and exosomes are applied as gene transporters to modify aberrant genes; (B) exosomes in the blood of a pregnant woman, amniotic fluid, or placenta are analyzed to diagnose cleft lips and palate; (C) exosomes can be used as drug or gene transporters to treat CLP by binding with a specific target, which increases the accuracy and treatment. CLP, cleft lip with palate.

showed that the downregulation of miR-300 and miR299-5p in amniotic fluid-based exosomes could serve as biomarkers for the diagnosis of congenital obstructive nephropathy (67). Recently, scientists have successfully used engineered exosomes containing an inhibitor of NF$\kappa \mathrm{B}$, a proinflammatory transcription factor whose activation stimulates preterm birth, to prolong gestation days and reduce maternal inflammation in a lipopolysaccharidechallenged mouse model (68). Exosomal biomarkers could also be used to detect and predict disorders in pregnant women and fetuses through urinary and blood exosomes affected by maternal abnormalities during gestation (69). A combination of type-B ultrasonic examination and plasma exosome detection could lead to more precise diagnoses and enable congenital malformations or fetal growth restrictions to be monitored before parturition.

The innate characteristics of exosomes suggest that they could be used as therapeutic agents. Their nanoscale size, immunocompatibility, nontoxicity, and access to biological barriers render them novel cell-free therapeutic instruments in disease treatments (see Figure 4). Exosomes can be engineered to carry specified cargos and synthetic cargos for therapy; for example, selected siRNAs, miRNAs, drugs, and enzymes can be loaded into exosomes directly by electroporation, chemical-based transfection, and simple incubation methods (3). Surface protein expression on exosomes could also be engineered to select cells and direct exosomes to specific regions (70). Exosomes from parental cells engineered to express an epidermal growth factor receptor (EGFR)-specific peptide ligand were successfully targeted to xenograft $\mathrm{EGFR}^{+}$breast cancer cells in mice (71), which suggests that the targeting of exosomes to cells could be accomplished by engineering the protein on lipid bilayers. The data obtained in a previous study show that exosomes isolated from pregnant women's plasma and serum contain not only maternal but also fetal-derived DNA. Exosomal miRNAs can enter the maternal blood and milk through the placenta or enter the amniotic fluid from the mother through the placenta. During pregnancy, the composition and content of exosomal miRNAs can change with mother's different statuses. The miRNAs of amniotic fluid exosomes are involved in the process of fertilized egg implantation, endometrial storage of fertilized eggs and placental function. Amniotic fluid exosomal miRNAs may be related to the 


\section{Page 10 of 13}

normal growth of the nervous system and other organs through synapses, synaptosomes, cell adhesion and chromatin activation protease pathways. In addition, miRNAs in amniotic fluid exosomes may be involved in the remodeling of fetal chromaticity. Currently, exosomes in blood are new noninvasive prenatal biomarkers in cancers, and they can not only predict risk but also be used as a potential therapy. Our wish is to diagnose nonsyndromic cleft lip and palate early with new noninvasive prenatal biomarkers.

In the past decade, a multitude of companies have been established to boost the biotechnology development of exosomes. Codiak BioSciences, a pioneer biotechnology company that has developed exosomal treatments for various diseases, developed the engEx ${ }^{\mathrm{TM}}$ Platform to engineer exosomes to express and deliver therapeutic drug candidates (72). The ExoDx Prostate ${ }^{\circledR}$ (IntelliScore) (EPI) test has been applied to assess patients with a risk of highgrade prostate cancer (73). Additionally, Avalon GloboCare established and developed the first saliva-based exosome miRNA biomarker, miR-185, as a dual target for the diagnosis and treatment of oral cancer.

Despite current challenges, the use of exosomes as diagnostic and therapeutic tools is promising and inspiring. Novel applications of exosomes in clinical practice can be expected in the near future.

\section{Conclusions and perspectives}

Over the past 20 years, the discovery of exosomes has made a revolutionary contribution to cell biology research. Exosomes are produced by most types of cells, circulate in body fluids, contain a variety of informational substances, can accurately transmit cellular signals, and can participate in a variety of physiological and pathological processes. Given the various types and limited amounts of genetic material in exosomes, methods for accurately selecting, maximizing the utility of, normalizing and quantifying exosomes urgently need to be developed. Exosomes mediate intercellular communication by specifically and selectively delivering biologically active molecules. However, the mechanism by which these molecules are synthesized and selectively packaged into exosomes is unknown, and further research needs to be conducted to extend the understanding of their mechanistic details. Exosomes can be applied in the treatment of CL/P by delivering therapeutic factors (e.g., RNA, proteins, or drugs) to target cells through genetic engineering approaches. It is assumed that exosomes need to be able to accurately locate their
Chen et al. Exosomes and cleft lip and palate: a narrative review

target cells and release their internal contents. However, the molecular mechanisms of exosomes in specific cells or tissues are far from clear. With an improved understanding of the etiology of CL/P, more efforts should be directed toward the clinical application of exosomal ncRNAs. By discovering and identifying genes and pathways related to $\mathrm{CL} / \mathrm{P}$ and improving the understanding of exosome-related pathogenesis, we can determine whether the abnormal genetic materials contained in exosomes are present in amniotic fluid and placental cells and deliver drugs or genes to target cells by exosomes to repair the mutated genes in a timely manner to prevent adverse effects. It is our view that exosomes will become a valuable tool for the diagnosis and treatment of $\mathrm{CL} / \mathrm{P}$ in the near future.

\section{Acknowledgments}

Funding: None.

\section{Footnote}

Reporting Checklist: The authors have completed the Narrative Review reporting checklist. Available at https:// dx.doi.org/10.21037/atm-21-4198

Conflicts of Interest: All authors have completed the ICMJE uniform disclosure form (available at https://dx.doi. org/10.21037/atm-21-4198). The authors have no conflicts of interest to declare.

Ethical Statement: The authors are accountable for all aspects of this work, including ensuring that any questions related to the accuracy or integrity of any part of the work have been appropriately investigated and resolved.

Open Access Statement: This is an Open Access article distributed in accordance with the Creative Commons Attribution-NonCommercial-NoDerivs 4.0 International License (CC BY-NC-ND 4.0), which permits the noncommercial replication and distribution of the article with the strict proviso that no changes or edits are made and the original work is properly cited (including links to both the formal publication through the relevant DOI and the license). See: https://creativecommons.org/licenses/by-nc-nd/4.0/.

\section{References}

1. Manolio TA, Collins FS, Cox NJ, et al. Finding the 
missing heritability of complex diseases. Nature 2009;461:747-53.

2. Wehby GL, Murray JC. Folic acid and orofacial clefts: a review of the evidence. Oral Dis 2010;16:11-9.

3. Arenaccio C, Chiozzini C, Ferrantelli F, et al. Exosomes in Therapy: Engineering, Pharmacokinetics and Future Applications. Curr Drug Targets 2019;20:87-95.

4. Yan F, Dai Y, Iwata J, et al. An integrative, genomic, transcriptomic and network-assisted study to identify genes associated with human cleft lip with or without cleft palate. BMC Med Genomics 2020;13:39.

5. Jugessur A, Farlie PG, Kilpatrick N. The genetics of isolated orofacial clefts: from genotypes to subphenotypes. Oral Dis 2009;15:437-53.

6. Murray JC. Gene/environment causes of cleft lip and/or palate. Clin Genet 2002;61:248-56.

7. Marazita ML, Murray JC, Lidral AC, et al. Meta-analysis of 13 genome scans reveals multiple cleft lip/palate genes with novel loci on 9q21 and 2q32-35. Am J Hum Genet 2004;75:161-73.

8. Beaty TH, Murray JC, Marazita ML, et al. A genomewide association study of cleft lip with and without cleft palate identifies risk variants near MAFB and ABCA4. Nat Genet 2010;42:525-9.

9. Reynolds K, Zhang S, Sun B, et al. Genetics and signaling mechanisms of orofacial clefts. Birth Defects Res 2020;112:1588-634.

10. Younkin SG, Scharpf RB, Schwender H, et al. A genomewide study of inherited deletions identified two regions associated with nonsyndromic isolated oral clefts. Birth Defects Res A Clin Mol Teratol 2015;103:276-83.

11. Bush JO, Jiang R. Palatogenesis: morphogenetic and molecular mechanisms of secondary palate development. Development 2012;139:231-43.

12. Hammond NL, Dixon J, Dixon MJ. Periderm: Life-cycle and function during orofacial and epidermal development. Semin Cell Dev Biol 2019;91:75-83.

13. Zhou J, Gao Y, Lan Y, et al. Pax9 regulates a molecular network involving Bmp4, Fgf10, Shh signaling and the Osr2 transcription factor to control palate morphogenesis. Development 2013;140:4709-18.

14. Smith TM, Lozanoff S, Iyyanar PP, et al. Molecular signaling along the anterior-posterior axis of early palate development. Front Physiol 2013;3:488.

15. Jin JZ, Ding J. Analysis of cell migration, transdifferentiation and apoptosis during mouse secondary palate fusion. Development 2006;133:3341-7.

16. Chen Q, Ding X, Lei J, et al. Comparison of the biological behaviors of palatal mesenchymal and epithelial cells induced by 2,3,7,8-tetrachlorodibenzo-p-dioxin in vitro. Toxicol Lett 2020;333:90-6.

17. Cuervo R, Covarrubias L. Death is the major fate of medial edge epithelial cells and the cause of basal lamina degradation during palatogenesis. Development 2004;131:15-24.

18. Aoyama G, Kurosaka H, Oka A, et al. Observation of Dynamic Cellular Migration of the Medial Edge Epithelium of the Palatal Shelf in vitro. Front Physiol 2019;10:698.

19. Li C, Lan Y, Jiang R. Molecular and Cellular Mechanisms of Palate Development. J Dent Res 2017;96:1184-91.

20. Lan Y, Jiang R. Sonic hedgehog signaling regulates reciprocal epithelial-mesenchymal interactions controlling palatal outgrowth. Development 2009;136:1387-96.

21. Rice R, Spencer-Dene B, Connor EC, et al. Disruption of Fgf10/Fgfr2b-coordinated epithelial-mesenchymal interactions causes cleft palate. J Clin Invest 2004;113:1692-700.

22. Chai Y, Maxson RE Jr. Recent advances in craniofacial morphogenesis. Dev Dyn 2006;235:2353-75.

23. Weng M, Chen Z, Xiao Q, et al. A review of FGF signaling in palate development. Biomed Pharmacother 2018;103:240-7.

24. Beermann J, Piccoli MT, Viereck J, et al. Non-coding RNAs in Development and Disease: Background, Mechanisms, and Therapeutic Approaches. Physiol Rev 2016;96:1297-325.

25. Peng Y, Croce CM. The role of MicroRNAs in human cancer. Signal Transduct Target Ther 2016;1:15004.

26. Wang Y, Medvid R, Melton C, et al. DGCR8 is essential for microRNA biogenesis and silencing of embryonic stem cell self-renewal. Nat Genet 2007;39:380-5.

27. Spruce T, Pernaute B, Di-Gregorio A, et al. An early developmental role for miRNAs in the maintenance of extraembryonic stem cells in the mouse embryo. Dev Cell 2010;19:207-19.

28. Li J, Zou J, Li Q, et al. Assessment of differentially expressed plasma microRNAs in nonsyndromic cleft palate and nonsyndromic cleft lip with cleft palate. Oncotarget 2016;7:86266-79.

29. Li A, Jia P, Mallik S, et al. Critical microRNAs and regulatory motifs in cleft palate identified by a conserved miRNA-TF-gene network approach in humans and mice. Brief Bioinform 2020;21:1465-78.

30. Shin JO, Lee JM, Cho KW, et al. MiR-200b is involved in Tgf- $\beta$ signaling to regulate mammalian palate 


\section{Page 12 of 13}

development. Histochem Cell Biol 2012;137:67-78.

31. Wang J, Bai Y, Li H, et al. MicroRNA-17-92, a direct Ap$2 \alpha$ transcriptional target, modulates T-box factor activity in orofacial clefting. PLoS Genet 2013;9:e1003785.

32. Li L, Shi JY, Zhu GQ, et al. MiR-17-92 cluster regulates cell proliferation and collagen synthesis by targeting TGFB pathway in mouse palatal mesenchymal cells. J Cell Biochem 2012;113:1235-44.

33. Zhang W, Shen Z, Xing Y, et al. MiR-106a-5p modulates apoptosis and metabonomics changes by TGF- $\beta /$ Smad signaling pathway in cleft palate. Exp Cell Res 2020;386:111734.

34. Ulitsky I, Bartel DP. lincRNAs: genomics, evolution, and mechanisms. Cell 2013;154:26-46.

35. Quinn JJ, Chang HY. Unique features of long noncoding RNA biogenesis and function. Nat Rev Genet 2016;17:47-62.

36. Peng $\mathrm{Z}$, Liu $\mathrm{C}, \mathrm{Wu} \mathrm{M}$. New insights into long noncoding RNAs and their roles in glioma. Mol Cancer 2018;17:61.

37. Salmena L, Poliseno L, Tay Y, et al. A ceRNA hypothesis: the Rosetta Stone of a hidden RNA language? Cell 2011;146:353-8.

38. Shu $X$, Dong $Z$, Zhang $M$, et al. Integrated analysis identifying long non-coding RNAs (lncRNAs) for competing endogenous RNAs (ceRNAs) networkregulated palatal shelf fusion in the development of mouse cleft palate. Ann Transl Med 2019;7:762.

39. Gao LY, Hao XL, Zhang L, et al. Identification and characterization of differentially expressed lncRNA in 2,3,7,8-tetrachlorodibenzo-p-dioxin-induced cleft palate. Hum Exp Toxicol 2020;39:748-61.

40. Liang WC, Fu WM, Wong CW, et al. The lncRNA H19 promotes epithelial to mesenchymal transition by functioning as miRNA sponges in colorectal cancer. Oncotarget 2015;6:22513-25.

41. Greene J, Baird AM, Brady L, et al. Circular RNAs: Biogenesis, Function and Role in Human Diseases. Front Mol Biosci 2017;4:38.

42. Conn SJ, Pillman KA, Toubia J, et al. The RNA binding protein quaking regulates formation of circRNAs. Cell 2015;160:1125-34.

43. Shu X, Cheng L, Dong Z, et al. Identification of circular RNA-associated competing endogenous RNA network in the development of cleft palate. J Cell Biochem 2019;120:16062-74.

44. Wapner RJ, Babiarz JE, Levy B, et al. Expanding the scope of noninvasive prenatal testing: detection of fetal microdeletion syndromes. Am J Obstet Gynecol

\section{Chen et al. Exosomes and cleft lip and palate: a narrative review}

2015;212:332.e1-9.

45. Bianchi DW. From prenatal genomic diagnosis to fetal personalized medicine: progress and challenges. Nat Med 2012;18:1041-51.

46. Lord J, McMullan DJ, Eberhardt RY, et al. Prenatal exome sequencing analysis in fetal structural anomalies detected by ultrasonography (PAGE): a cohort study. Lancet 2019;393:747-57.

47. Oliver JD, Turner EC, Halpern LR, et al. Molecular Diagnostics and In Utero Therapeutics for Orofacial Clefts. J Dent Res 2020;99:1221-7.

48. Dugger SA, Platt A, Goldstein DB. Drug development in the era of precision medicine. Nat Rev Drug Discov 2018;17:183-96.

49. Gurunathan S, Kang MH, Jeyaraj M, et al. Review of the Isolation, Characterization, Biological Function, and Multifarious Therapeutic Approaches of Exosomes. Cells 2019;8:307.

50. Shao H, Im H, Castro CM, et al. New Technologies for Analysis of Extracellular Vesicles. Chem Rev 2018;118:1917-50.

51. Pegtel DM, Gould SJ. Exosomes. Annu Rev Biochem 2019;88:487-514.

52. Kim KM, Abdelmohsen K, Mustapic M, et al. RNA in extracellular vesicles. Wiley Interdiscip Rev RNA 2017. doi: 10.1002/wrna.1413.

53. Xie F, Zhou X, Fang M, et al. Extracellular Vesicles in Cancer Immune Microenvironment and Cancer Immunotherapy. Adv Sci (Weinh) 2019;6:1901779.

54. Zhang R, Xia Y, Wang Z, et al. Serum long non coding RNA MALAT-1 protected by exosomes is up-regulated and promotes cell proliferation and migration in nonsmall cell lung cancer. Biochem Biophys Res Commun 2017;490:406-14.

55. Mittelbrunn M, Gutiérrez-Vázquez C, Villarroya-Beltri $\mathrm{C}$, et al. Unidirectional transfer of microRNA-loaded exosomes from $\mathrm{T}$ cells to antigen-presenting cells. Nat Commun 2011;2:282.

56. Dai J, Su Y, Zhong S, et al. Exosomes: key players in cancer and potential therapeutic strategy. Signal Transduct Target Ther 2020;5:145.

57. Li Z, Jiang P, Li J, et al. Tumor-derived exosomal lncSox2ot promotes EMT and stemness by acting as a ceRNA in pancreatic ductal adenocarcinoma. Oncogene 2018;37:3822-38.

58. Campos A, Salomon C, Bustos R, et al. Caveolin-1containing extracellular vesicles transport adhesion proteins and promote malignancy in breast cancer cell 
lines. Nanomedicine (Lond) 2018;13:2597-609.

59. Qu Z, Feng J, Pan H, et al. Exosomes derived from HCC cells with different invasion characteristics mediated EMT through TGF- $\beta /$ Smad signaling pathway. Onco Targets Ther 2019;12:6897-905.

60. Zhang X, Sai B, Wang F, et al. Hypoxic BMSC-derived exosomal miRNAs promote metastasis of lung cancer cells via STAT3-induced EMT. Mol Cancer 2019;18:40.

61. Zlotogorski-Hurvitz A, Dayan D, Chaushu G, et al. Morphological and molecular features of oral fluid-derived exosomes: oral cancer patients versus healthy individuals. J Cancer Res Clin Oncol 2016;142:101-10.

62. Jiang N, Xiang L, He L, et al. Exosomes Mediate Epithelium-Mesenchyme Crosstalk in Organ Development. ACS Nano 2017;11:7736-46.

63. Hayashi T, Lombaert IM, Hauser BR, et al. Exosomal MicroRNA Transport from Salivary Mesenchyme Regulates Epithelial Progenitor Expansion during Organogenesis. Dev Cell 2017;40:95-103.

64. Tobón-Arroyave SI, Celis-Mejía N, Córdoba-Hidalgo MP, et al. Decreased salivary concentration of CD9 and CD81 exosome-related tetraspanins may be associated with the periodontal clinical status. J Clin Periodontol 2019;46:470-80.

65. Huang X, Hu X, Zhao M, et al. Analysis of salivary exosomal proteins in young adults with severe periodontitis. Oral Dis 2020;26:173-81.

66. Xing X, Han S, Li Z, et al. Emerging role of exosomes

Cite this article as: Chen M, Xie Y, Luo Y, Xie Y, Wu N, Peng S, Chen Q. Exosomes-a potential indicator and mediator of cleft lip and palate: a narrative review. Ann Transl Med 2021;9(18):1485. doi: 10.21037/atm-21-4198 in craniofacial and dental applications. Theranostics 2020;10:8648-64.

67. Xie J, Zhou Y, Gao W, et al. The relationship between amniotic fluid miRNAs and congenital obstructive nephropathy. Am J Transl Res 2017;9:1754-63.

68. Sheller-Miller S, Radnaa E, Yoo JK, et al. Exosomal delivery of NF- $\kappa$ B inhibitor delays LPS-induced preterm birth and modulates fetal immune cell profile in mouse models. Sci Adv 2021;7:eabd3865.

69. Jiang PY, Zhu XJ, Jiang RA, et al. MicroRNAs derived from urinary exosomes act as novel biomarkers in the diagnosis of intrahepatic cholestasis of pregnancy. Am J Transl Res 2019;11:6249-61.

70. Cooper LF, Ravindran S, Huang CC, et al. A Role for Exosomes in Craniofacial Tissue Engineering and Regeneration. Front Physiol 2020;10:1569.

71. Ohno S, Takanashi M, Sudo K, et al. Systemically injected exosomes targeted to EGFR deliver antitumor microRNA to breast cancer cells. Mol Ther 2013;21:185-91.

72. Zhou B, Xu K, Zheng X, et al. Application of exosomes as liquid biopsy in clinical diagnosis. Signal Transduct Target Ther 2020;5:144.

73. McKiernan J, Donovan MJ, Margolis E, et al. A Prospective Adaptive Utility Trial to Validate Performance of a Novel Urine Exosome Gene Expression Assay to Predict High-grade Prostate Cancer in Patients with Prostate-specific Antigen 2-10ng/ml at Initial Biopsy. Eur Urol 2018;74:731-8. 